\title{
CSF1 Expression in Nongynecological Leiomyosarcoma Is Associated with Increased Tumor Angiogenesis
}

\author{
Iñigo Espinosa, ${ }^{* \dagger}$ Badreddin Edris, ${ }^{* \neq}$ \\ Cheng-Han Lee, ${ }^{\S}$ Hong Wei Cheng, ${ }^{\S}$ \\ C. Blake Gilks, ${ }^{\S}$ Yuzhuo Wang," \\ Kelli D. Montgomery, ${ }^{*}$ Sushama Varma, ${ }^{*}$ Rui Li, ${ }^{*}$ \\ Robert J. Marinelli," Robert B. West, ${ }^{*}$ \\ Torsten Nielsen, ${ }^{\S}$ Andrew H. Beck, ${ }^{*}$ and \\ Matt van de Rijn*
}

\begin{abstract}
From the Department of Pathology,* Stanford University Medical Center, Stanford, California; the Department of Pathology, ${ }^{\dagger}$ Hospital de la Santa Creu i Sant Pau, Institute of Biomedical Research (IIB Sant Pau), Autonomous University of Barcelona, Barcelona, Spain; the Departments of Genetics, ${ }^{\ddagger}$ and Biochemistry," Stanford University School of Medicine, Stanford, California; the Department of Pathology, University of British Columbia, Vancouver, British Columbia, Canada; and the British Columbia Cancer Agency, "Vancouver, British Columbia, Canada
\end{abstract}

Leiomyosarcoma (LMS) is a malignant tumor of smooth muscle cells for which few effective therapies exist. A subset of LMS cases express macrophage colony-stimulating factor (CSF1) and the resultant tumor-associated macrophage (TAM) infiltration predicts poor clinical outcome. Further, TAMs have been shown to increase tumor angiogenesis. Here, we analyzed 149 LMS cases by immunohistochemistry for vascular marker CD34 and show that high microvessel density (MVD) in nongynecological LMS cases significantly predicts poor patient outcome. The majority of high MVD cases were also CSF1-positive, and when combining high MVD with CSF1 expression, an even stronger prognostic correlation with patient outcome was obtained. Gene expression profiling revealed that MVD has a stronger correlation with CSF1 expression than with expression of vascular endothelial growth factor isoforms, which have traditionally been used as markers of angiogenesis and as antiangiogenic therapeutic targets. Finally, patterns of CSF1 expression and TAM recruitment remained consistent between primary tumors and their metastases, and between primary tumors and those grown as xenografts in mice, highlighting the stability of these features to the biology of LMS tumors. Together, these findings suggest an important role for CSF1 and the resulting TAM infiltration in the pathological neovascularization of LMS tumors and provide a rationale for CSF1-targeted therapies in LMS. (Am J Pathol 2011, 179: 2100-2107; DOI: 10.1016/j.ajpath.2011.06.021)

In carcinomas, the contributions of CSF1 production and macrophage recruitment/activation to tumor progression have been well-studied. Tumor associated macrophages (TAMs) have a wide range of activities and may promote tumor growth, angiogenesis, extracellular matrix breakdown, invasion, and metastasis. ${ }^{1-3}$ The formation of new blood vessels and the degree of tumor neovascularity has been correlated with the development of metastasis in several cancers, including those arising in the breast, prostate, ovary, lung, colon, skin, testis, and bladder. ${ }^{4-10}$ In fact, TAMs produce a variety of pro-angiogenic factors that include vascular endothelial growth factor (VEGF), basic fibroblast growth factor, tumor necrosis factor $\alpha$ (TNF $\alpha$ ), and others. In addition, several studies have shown an association between the numbers of TAMs and microvessel density (MVD). ${ }^{11-13}$

In carcinomas the tumor microenvironment (TME) is prominently visible on histological examination and consists of a complex mixture of a wide variety of cell types. The TME is less obvious by histological examination in most sarcomas ${ }^{14}$ and few studies have addressed the role of the TME in sarcomas. LMS are tumors of smooth

Supported in part by grants from the National Institutes of Health grant (CA112270), the National Leiomyosarcoma Foundation, and the Leiomyosarcoma Direct Research Foundation (M.v.d.R.).

Accepted for publication June 20, 2011

CME Disclosure: None of the authors disclosed any relevant financial relationships.

Supplemental material for this article can be found at http://ajp. amjpathol.org or at doi: 10.1016/j.ajpath.2011.06.021.

Address reprint requests to Matt van de Rijn, M.D., Ph.D., Department of Pathology, L-235, Stanford University Medical Center, 300 Pasteur Dr., Stanford, CA 94305. E-mail: mrijn@stanford.edu. 
muscle that can occur predominantly in the female genital tract (gynecologic LMS) or in the deep soft tissues (nongynecologic LMS). We have previously shown that the presence of CD163-, FCGR3a-, and CTSL1-positive macrophages is increased in LMS cases that express CSF1 in the tumor cells and that this predicts poor clinical outcome in both nongynecological and gynecological LMS. ${ }^{15,16}$ However, the mechanism by which macrophage infiltration in LMS affects clinical outcome remains to be elucidated. Previous studies found no correlation between MVD and clinical outcome in sarcoma. While these studies examined a wide range of sarcomas, they did not include large numbers of LMS. In this study, we address the correlation between CSF1, TAMs, MVD, and clinical outcome in LMS.

\section{Materials and Methods}

\section{Human Exonic Evidence Based Oligonucleotide Gene Arrays}

For gene expression analysis, we used a data set obtained from 16 LMS, which have been previously described. ${ }^{15,16}$ Our current analysis was restricted to CSF1, CSF1R, CD34, VEGFA, VEGFB, and VEGFC. Only cases with $>80 \%$ available good data for these genes were further analyzed.

\section{Tissue Microarray Construction}

Two tissue microarrays (TA 121 and 201) containing 149 primary LMS cases from 149 patients with clinical follow up were used in this study. For 13 cases, material from metastases was available. None of these patients received adjuvant therapy (chemotherapy and/or radiotherapy). The clinicopathological features of the 16 LMS used for gene expression studies and the 149 LMS used on the tissue microarray were previously described. ${ }^{15,16}$ Briefly, the 149 primary LMS consisted of 73 nongynecological and 76 gynecological cases. The nongynecological LMS group ( $n=73$ ) contained tumors from 42 females and 31 males with a median age of 54 years (range, 13 to 81 years) and a median tumor size of $9.9 \mathrm{~cm}$ (range, 1.2 to $34 \mathrm{~cm}$ ). Limbs and the retroperitoneum were the most common tumor locations. The median age of the women in the gynecological LMS group $(n=76)$ was 50.7 years (range, 5 to 67 years) and the median tumor size was $10.1 \mathrm{~cm}$ (range, 2 to $35 \mathrm{~cm}$ ). The uterus was by far the most common tumor site for gynecologic LMS. The mean follow-up time was 3.1 years with an overall range of 1 month to 5 years. Tissue microarrays were constructed using 0.6-mm cores with a tissue arrayer (Beecher Instruments, Silver Spring, MD). Gynecological LMS were classified according to tumor differentiation (ie, well, moderate, and poorly differentiated) and nongynecological LMS were staged by the Fédération Nationale des Centres de Lutte Contre le Cancer grading system. The diagnosis of LMS was based on morphological criteria and immunohistochemistry results as previously described. ${ }^{15,16}$

\section{Immunohistochemistry}

Slides were cut at $4 \mu \mathrm{m}$, deparaffinized in xylene and hydrated in a graded series of alcohol. For immunohistochemistry, CD34 (581, mouse monoclonal, 1/40; Becton Dickinson Biosciences, San Jose, CA) was the primary antibody used. Slides were boiled in antigen retrieval solution (citrate, $\mathrm{pH}$ 6) for 12 minutes. Murine macrophages were stained by rat anti-mouse macrophage F4/80 monoclonal antibody (BM8, 1/50, Invitrogen Corp, Carlsbad, CA). The immunohistochemical reactions were visualized using mouse versions of the EnVision + system (DAKO, Carpinteria, CA) using diaminobenzidine. MVD was determined by counting the number of CD34positive microvessels in an entire 0.6- $\mathrm{mm}$ core. A microvessel was defined as any endothelial cell or endothelial cell cluster staining positive for CD34. ${ }^{17}$ The definition of microvessels and their scoring was done before analysis of patient outcome data. Digital images from all stained cores are available through the Stanford tissue microarray database. ${ }^{18}$

\section{Xenograft Study}

Animal care and experiments were carried out in accordance with the guidelines of the Canadian Council on Animal Care and were approved by the Animal Care Committee of The University of British Columbia. For xenograft studies, approximately $1 \mathrm{~mL}$ of fresh tumor tissue was obtained from each surgical specimen, and trimmed fresh fragments ( 6 to $\sim 8 \mathrm{~mm}^{3}$ in size) were implanted underneath the capsule of kidneys exteriorized through dorsal incisions in two to three nonobese diabetic/severe combined immunodeficiency mice as previously described in detail. ${ }^{19,20}$ Xenografts were retrieved after 30 or 60 days (postgraft tissues) for confirmation of tumor histology and subsequent passages with transplant generations amounting to 30 or 60 days. Two high-grade uterine LMS were established as xenograft tumor lines (LMS \#11436 and LMS \#17411), and were carried to passage 5 .

\section{Statistical Analysis}

The Kaplan-Meier method was used to estimate diseasespecific survival (DSS) distributions and Log-rank (Mantel-Cox) tests were used to ascertain whether given variables significantly correlated with DSS. The DSS was calculated from the time of diagnosis to the date of death of the disease. For gene expression analysis, a Pearson correlation matrix was used to study the association between CSF1, CSF1R, CD34, VEGFA, VEGFB, and VEGFC. $P$ values $\leq 0.05$ were considered significant. Statistical calculations were performed using SPSS software v.14.0 (SPSS, Chicago, IL) and GraphPad Prism v5.0 (GraphPad Software, San Diego, CA). Hierarchical cluster analysis of TMA immunostaining results was realized using Deconvoluter 6 and TMA-Combiner 7 programs. ${ }^{21}$ 


\section{Results}

\section{Prognostic Significance of MVD in LMS}

To ascertain the density of microvessels in LMS tumors, two tissue microarrays containing 76 gynecologic and 73 nongynecologic primary LMS were stained with a CD34specific antibody, a marker for vessel endothelial cells; of the cases on the array, 71 of each subtype were evaluable for CD34 expression (Figures $1 \mathrm{~A}$ and 1B). There was considerable variation in MVD across the different cases of LMS. Microvessel count was higher in gynecological LMS, where it ranged from 0 to 140 per $0.6-\mathrm{mm}$ core (mean, 35 per 0.6-mm core), than in nongynecological
LMS, where it ranged from 0 to 100 per $0.6-\mathrm{mm}$ core (mean, 22 per 0.6-mm core) ( $t$-test; $P=0.0012$ ) (Figure 1C). Gynecological LMS also contained a higher proportion of tumors with high MVD (>40 microvessels/0.6 mm) than nongynecological LMS (Figure 1D).

Cases were stratified based on whether they displayed greater or less than the mean MVD for their respective subtypes, and DSS was used as an endpoint measurement for Kaplan-Meier analyses. In gynecological LMS, MVD showed no significant association with DSS $(n=71$; hazard ratio: $1.104 ; 95 \%$ confidence interval: 0.415 to 2.938; $P=0.8429$; Figure 1E). In contrast, a significant correlation between MVD and DSS was found in nongy-

\section{A CD34 IHC - Gynecological LMS}

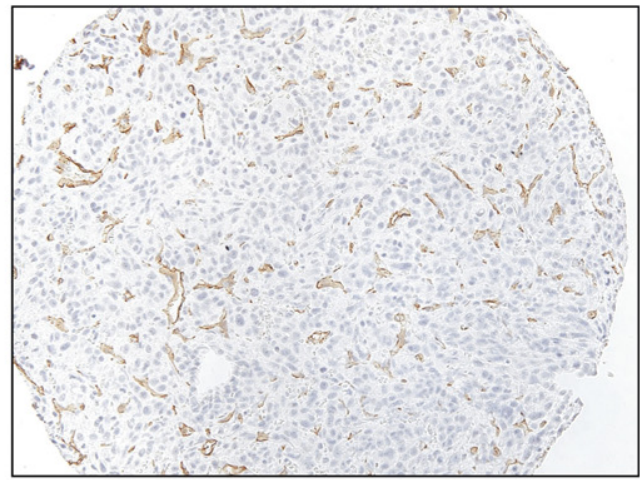

C

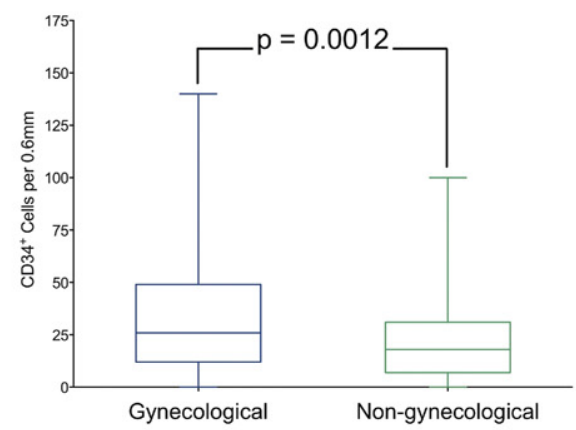

E

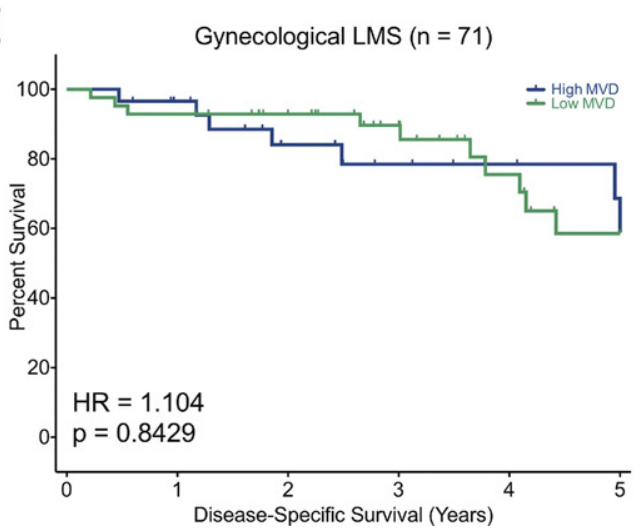

B
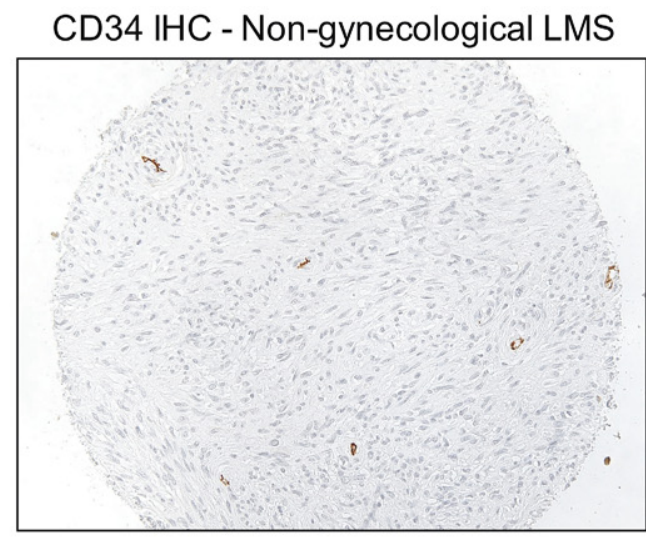

D

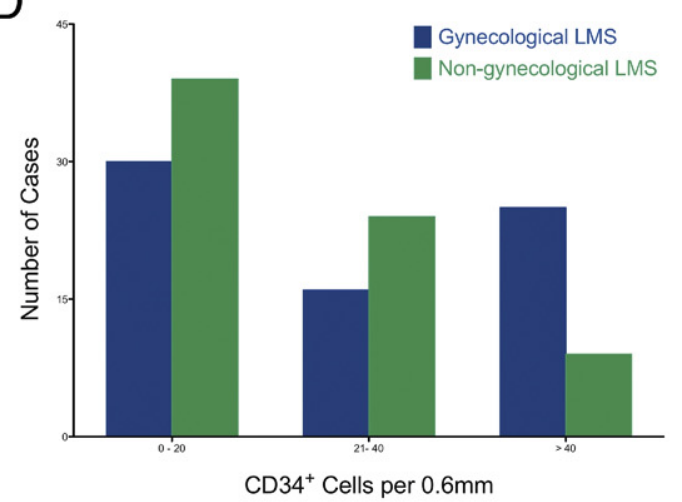

$\mathrm{F}$

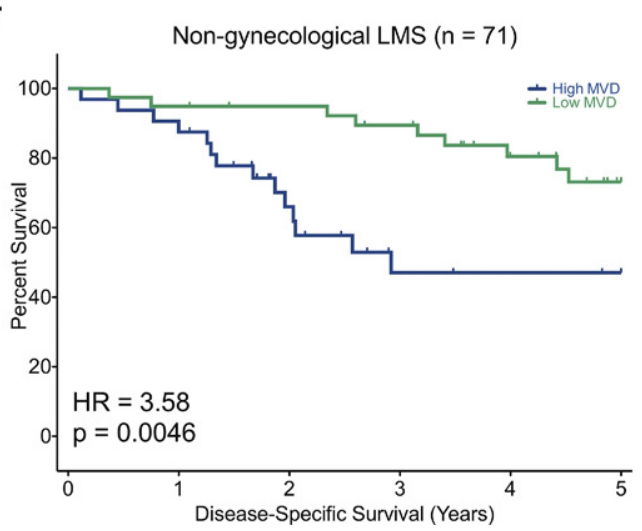

Figure 1. Representative CD34 stains in a gynecological (A) and nongynecological (B) leiomyosarcoma (LMS) case. Distribution of gynecological and nongynecological LMS cases based on microvessel density (MVD) in each 0.6-mm diameter TMA core (C, D). Correlation of MVD with disease-specific survival in gynecological (E) and nongynecological (F) LMS. HR, hazard ratio; IHC, immunohistochemistry. 


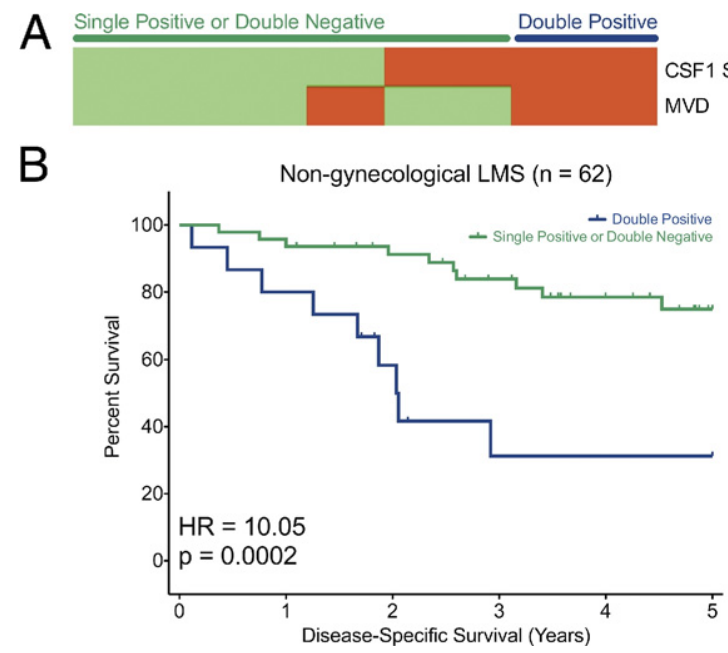

Figure 2. Nongynecological leiomyosarcoma (LMS) cases positive for the CSF1 signature (defined as coordinate expression of CSF1, CTSL1, FCGFR3a, and CD163) were more likely to show high microvessel density (MVD) than cases that were negative for the CSF1 signature (A). Stratification of patients based on high MVD and the presence of the CSF1 response is associated with even greater survival differences than when MVD was considered alone, as shown in (B). HR, hazard ratio.

necological LMS ( $n=71$; hazard ratio: $3.58 ; 95 \% \mathrm{Cl}: 1.48$ to $8.65 ; P=0.0046)$, thereby suggesting an important role for angiogenesis in these tumors (Figure 1F).

\section{High MVD LMS Cases Mark a Subset of CSF1-Positive Tumors}

Recently, it was shown that depletion of CSF1 resulted in the suppression of tumor angiogenesis in a mouse model of osteosarcoma, ${ }^{22}$ thereby highlighting the potential importance of this molecule in mediating angiogenesis in tumors. Given the known prognostic significance of CSF1 expression in LMS tumors, as well as the association of high MVD with decreased DSS in nongynecological LMS, we sought to determine the association between MVD and CSF1 expression in the nongynecological LMS cases. To do so, we used the CSF1 signature, which is defined as the coordinate expression in the tumor site of CSF1 (as measured by RNA in situ hybridization), CD163, FCGR3a, and CTSL1 (as measured by immunohistochemistry), which we previously showed predicts poor outcome in LMS. ${ }^{15}$ There were 62 nongynecological LMS cases that had both evaluable MVD and CSF1 signature data. In these tumors, CSF1 signature-positive cases were significantly more likely than CSF1 signature-negative cases to have increased tumor vascularity with 15 of $28(53 \%)$ CSF1-signature positive cases showing high MVD compared to only 8 of 34 (24\%) CSF1 signaturenegative cases showing high MVD (Fisher's exact test; $P=0.019$ ) (Figure 2A)

The 15 cases that were doubly positive for the CSF1 signature and for high MVD showed a very strong correlation with poor patient outcome when compared to the 47 cases that were either singly positive for the CSF1 signature or for high MVD, or negative for both (hazard ratio: $10.05 ; 95 \% \mathrm{Cl}: 2.944$ to $34.31 ; P=0.0002$ ), thereby highlighting the usefulness of using both the CSF1 signature and MVD to stratify patients into groups that show significant prognostic differences (Figure 2B). Together, the significant association between CSF1 signature expression and MVD, as well as the increased prognostic association derived from using both features to predict patient outcome, suggest that CSF1 may be mediating an angiogenic phenotype in nongynecological LMS, and that this angiogenic phenotype is characteristic of a highly aggressive subset of LMS tumors.

\section{CSF1 Expression Shows a Stronger Correlation with Tumor Vascularization than Does VEGF in Nongynecological LMS}

The roles of VEGFA, VEGFB, and VEGFC in mediating tumor vascularization have been well documented and have formed the rationale for targeting these VEGF isoforms therapeutically in cancer treatment. Given the role of these molecules in pathological microvessel formation, we sought to compare their correlation with MVD to that of CSF1 by performing a gene expression analysis of 16 LMS tumors (see Supplemental Figure S1 at http:// ajp.amjpathol.org). Interestingly, after performing pairwise Pearson correlations between mRNA levels for CSF1, $C D 34$, and VEGF genes, we found a significant positive correlation existed between the mRNA levels of CD34 and CSF1 ( $r=0.32$ ). In contrast, we found either no significant or negative correlations between CD34 and VEGFA, VEFGB, or VEGFC $(r=-0.10 ; r=-0.16 ; r=$ 0.17 , respectively) (Table 1 and Figure 3; see also Supplemental Table S1 at http://ajp.amjpathol.org). These results further suggest that CSF1 plays a significant role in pathological tumor vascularization in nongynecological LMS.

\section{CSF1 Expression, Macrophage Infiltration, and MVD Are Stable Properties of LMS Tumors}

We next examined the consistency in the pattern of CSF1 expression, macrophage recruitment, and MVD by comparing these features in 13 primary nongynecological LMS samples and their corresponding metastatic lesions. We found that 8 of $9(89 \%)$ tumors with high CSF1 ex-

Table 1. Pearson Correlation Coefficients of Markers within LMS

\begin{tabular}{lrrrrrr}
\hline & CD34 & CSF1 & CSF1R & VEGFC & VEGFB & VEGFA \\
\hline CD34 & 1.00 & & & & & \\
CSF1 & 0.32 & 1.00 & & & & \\
CSF1R & $0.6^{*}$ & $0.73^{*}$ & 1.00 & & & \\
VEGFC & 0.17 & $0.56^{*}$ & 0.28 & 1.00 & & \\
VEGFB & -0.16 & 0.01 & -0.27 & 0.03 & 1.00 & \\
VEGFA & -0.10 & -0.17 & -0.33 & -0.17 & 0.49 & 1.00 \\
\hline
\end{tabular}

Pearson pairwise comparisons matrix showing the correlations between the mRNA levels of CSF1, CSF1R, CD34, VEGFA, VEGFB, and VEGFC, as determined from Human Exonic Evidence Based Oligonucleotide gene array data on 16 LMS cases.

Statistically significant at ${ }^{*} P<0.05$

LMS, leiomyosarcoma; VEGF, vascular endothelial growth factor. 


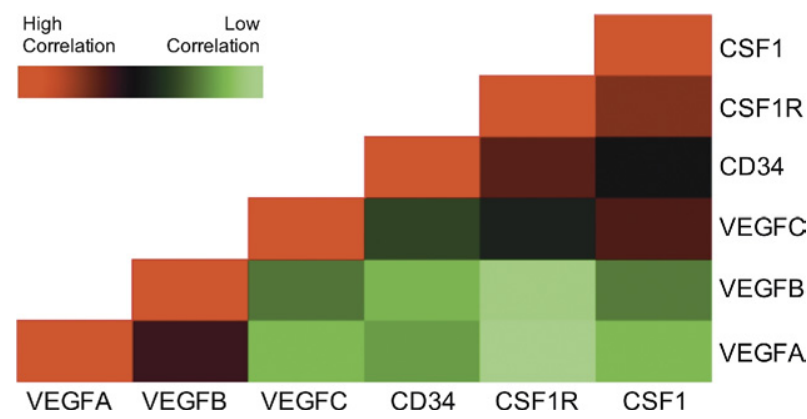

Figure 3. Heat map representing a Pearson correlation matrix generated from Human Exonic Evidence Based Oligonucleotide gene array expression data on 16 nongynecological leiomyosarcoma (LMS) cases displaying the correlations between the mRNA levels of CSF1, CSF1R, CD34, vascular endothelial growth factor (VEGF)A, VEGFB, and VEGFC. Red indicates a high correlation; black indicates an intermediate correlation; and green indicates a low correlation.

pression maintained this feature in their associated metastases. In addition, only $25 \%$ ( 1 of 4 ) of primary tumors that were negative for CSF1 showed expression of this molecule in their metastatic lesions (Figure 4A). The presence of TAMs, as measured by CD163 staining, also remained stable between primary and metastatic tumors. The majority of tumors ( 8 of $9 ; 88 \%$ ) with high expression of CD163 also showed large numbers of macrophages in their associated metastases. Likewise, all primary tumors with low expression of CD163 $(n=4)$, had a very low number of tumor macrophages in their corresponding metastases (Figure 4B). Finally, the level of MVD seen in the primary tumor remained similar in the corresponding metastases in 9 of 12 cases (Figure 4C).

To further examine whether CSF1 expression and infiltration by TAMs are intrinsic properties of LMS tumors, we evaluated two primary LMS in a mouse xenograft model of LMS. The parental tumor from one case (LMS \#17411) showed high CSF1 expression and a high density of CD163-positive stromal macrophage infiltrates, whereas the parental tumor from the second case (LMS \#11436) showed no CSF1 expression and very sparse CD163positive stromal macrophage infiltrates (Figure 5). Each tumor was transplanted into nonobese diabetic/severe combined immunodeficiency mice and was serially passaged into new mice up to passage 5. After 4 passages, no residual human macrophages were present in either case (data not shown). Remarkably, LMS \#17411 maintained its high CSF1 expression (data not shown) and displayed a higher density of host murine F4/80-positive macrophages as compared to the tumor derived from LMS \#11436 (Figure 5). These results further underscore the intrinsic nature of CSF1 expression and resultant macrophage infiltration in LMS tumors, lending additional strength to the observation that CSF1 expression and its consequences are maintained between primary and metastatic LMS tumors.

\section{Discussion}

The TME has been recognized as an active participant in tumor behavior in a wide range of epithelial malignan- cies, ${ }^{23,24}$ but few studies have addressed the role of the microenvironment in sarcomas. Here we have analyzed the role of tumor angiogenesis in LMS and its possible relationship with CSF1 expression.

The density of microvessels was predictive of outcome in nongynecologic LMS, but not in gynecologic LMS. The reason for this distinction is not clear, but the average number of microvessels in gynecologic LMS is significantly higher than that found in LMS originating in the soft tissues, perhaps related to the fact that the uterus (the most common site of origin for gynecologic LMS) is a highly vascularized organ. It is plausible that an increase in microvessels in a subset of gynecologic LMS may not have the same noticeable effect on outcome as that seen for LMS originating outside the female genital tract. Previous studies suggested that MVD was not prognostic of clinical outcome in soft-tissue sarcomas. ${ }^{25-28}$ In contrast

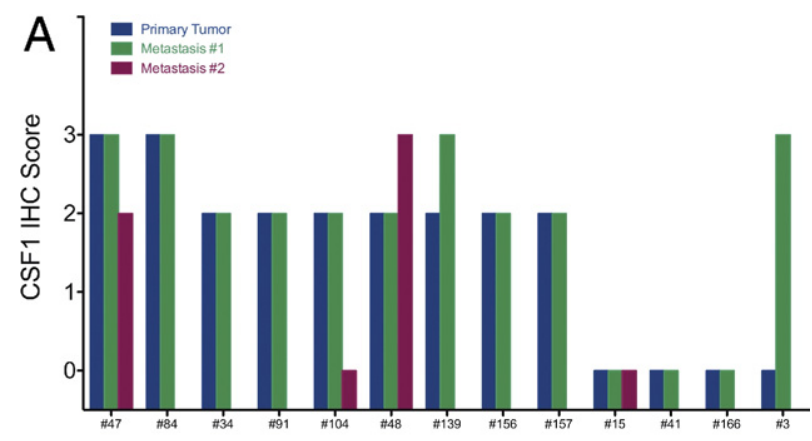

LMS Case ID Number

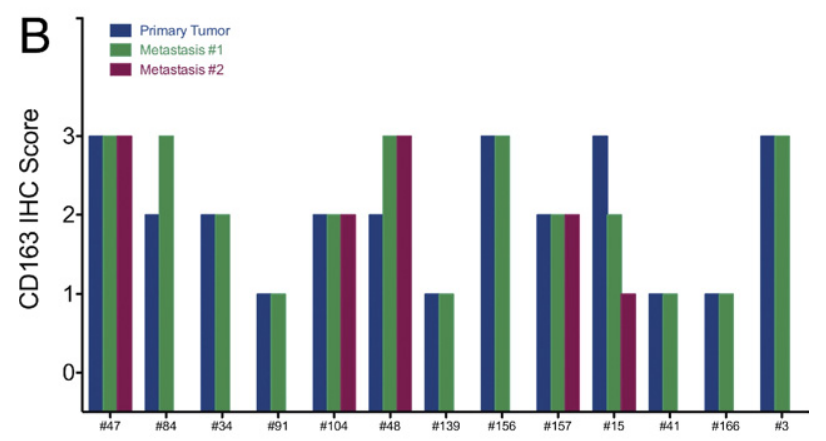

LMS Case ID Number

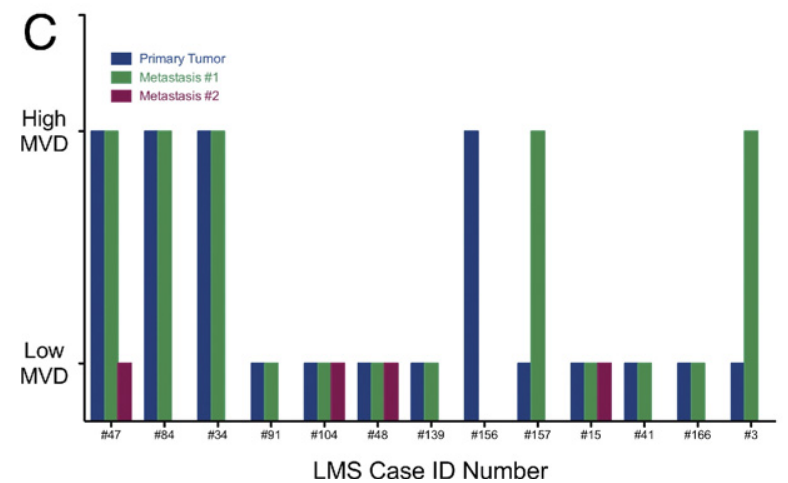

Figure 4. Maintenance of CSF1 expression (A), presence of CD163-positive tumor-associated macrophages (B), and microvessel density (MVD) (C) in primary leiomyosarcoma (LMS) tumors and their associated metastatic lesions. Numbers along the $x$ axis represent LMS case identifications. IHC, immunohistochemistry. 
Patients \#17411
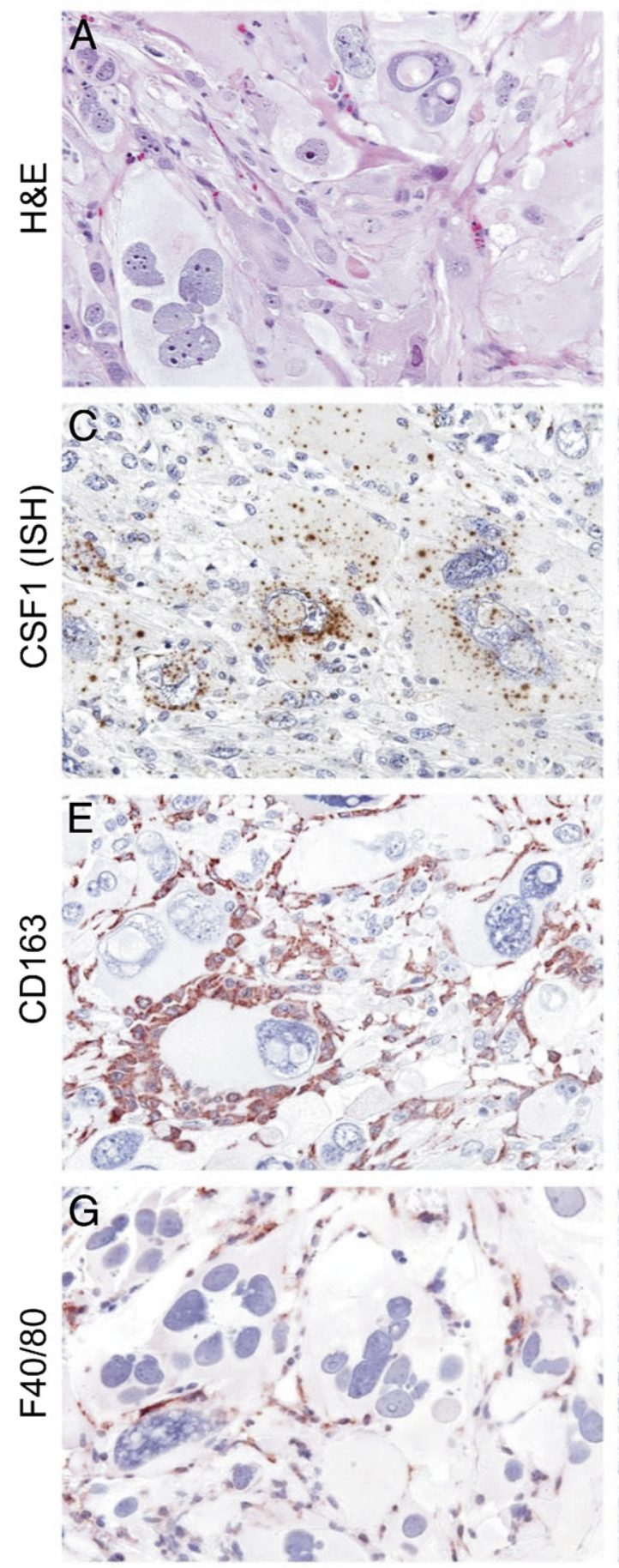

Patients \#11436
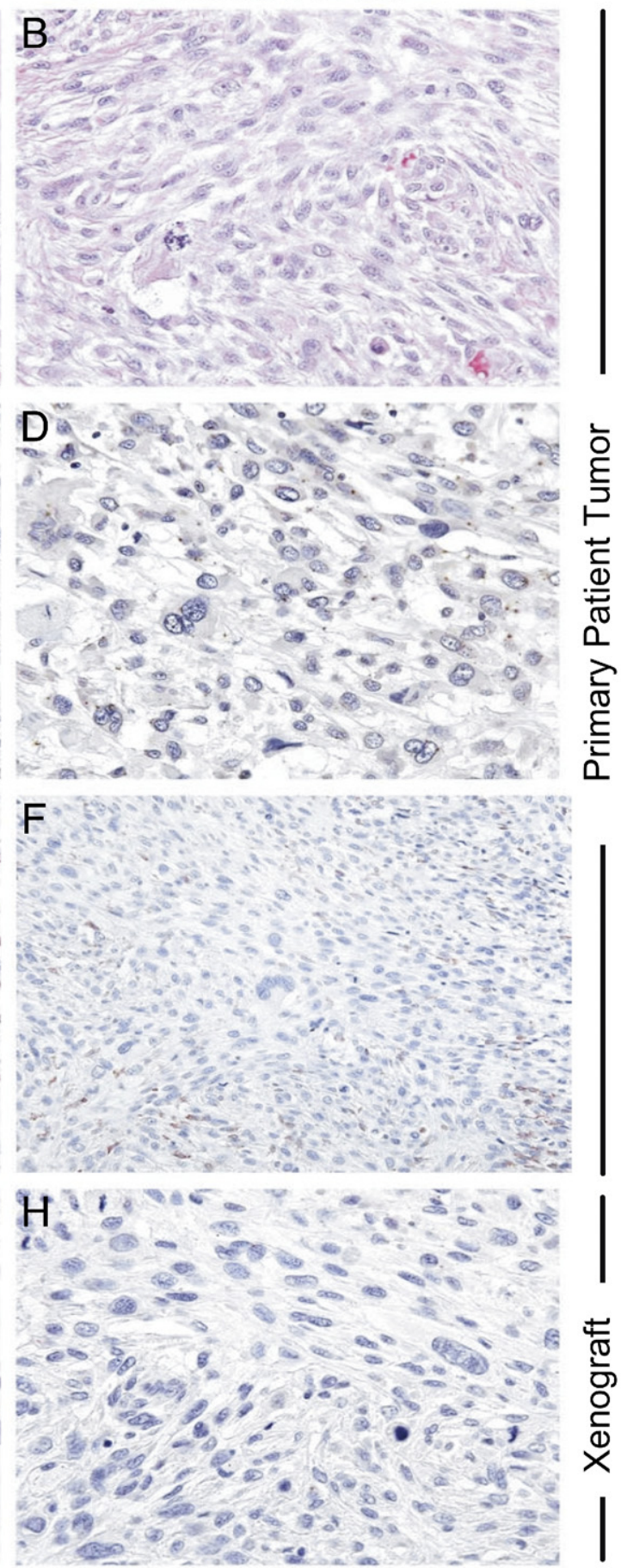

Figure 5. H\&E stains of two primary leiomyosarcoma (LMS) samples (A, B), CSF1 expression as measured by in situ hybridization (C, D), and infiltration by tumor-associated macrophages, as measured by CD163 immunohistochemistry (IHC) (E, F). After xenotransplantation into immunocompromised mice and five serial passages, the tumor derived from patient \#17411 shows high murine macrophage infiltration, as measured by IHC for mouse macrophage marker F $40 / 80$ (G). In contrast, the xenograft derived from patient \#11436 is unable to attract murine macrophages (H). ISH, in situ hybridization.

to our study, these investigations examined MVD in softtissue sarcomas without making distinctions between diagnostic subtypes. This lack of separation of individual sarcoma types, combined with the small number of LMS cases examined $(8,12$, and 18 , respectively) may explain why the association between MVD and poor clinical prog- nosis in nongynecological LMS was not apparent in those studies.

Previously, we showed that variable levels of CSF1 expression are found in LMS tumor cells. Those cases that have high levels of CSF1 production create a TME that is rich in TAMs, and this is associated with a poor 
clinical outcome. ${ }^{15,16}$ Many studies have demonstrated that TAMs can mediate opposite effects based on their polarization, sometimes resulting in activity against tumors cells and at other times mediating tumor progression. TAMs exhibiting anti-tumor activity (M1, or classically-activated macrophages) can mediate their effects through eliciting specific anti-tumor adaptive immunity. In contrast, TAMs exhibiting pro-tumor activity (M2, or alternatively activated macrophages) suppress adaptive immunity and promote a wound healing-like $\mathrm{TME}^{29}$ in which angiogenic and tissue remodeling processes lead to tumor cell invasion and ultimately cancer progression. ${ }^{3}$ In line with our results, pro-angiogenic, M2-polarized TAMs likely predominate in nongynecological LMS.

This relationship between TAMs and angiogenesis has been implicated in the tumorigenesis of several carcinomas. Lin et al. ${ }^{11}$ showed that CSF1-associated macrophages are involved in an "angiogenic switch" that results in promoting tumor angiogenesis and tumor progression to malignancy in a mouse model of breast cancer. However, few studies have analyzed the association between CSF1, TAMs, and angiogenesis in sarcomas.

Here we show there is a correlation between CSF1 levels and MVD in nongynecological LMS. First, nearly all LMS cases with high MVD were part of the subset of cases with high CSF1 levels. Second, adding MVD as a variable to the previously identified "CSF1-signature" prognosticator increased the ability to identify cases with poor outcome. Finally, mRNA analysis showed a higher correlation between CSF1 levels and MVD than that found between VEGF levels and MVD. In a recent study, Kubota et $\mathrm{al}^{22}$ showed that CSF1-associated macrophages stimulate angiogenesis independently of VEGF in an osteosarcoma model, and that this angiogenic effect was absent in normal tissue. Although many studies have focused on the role of VEGF in tumor angiogenesis, our findings are concordant with the results obtained by Kubota et al. ${ }^{22}$ In their investigations, CSF1-dependent macrophages contributed to both vascular and lymphatic development; their results suggested that macrophagemediated extracellular matrix disorganization was mediated through high levels of matrix metalloproteinase-2 and matrix metalloproteinase-9 expression rather than being mediated through VEGF. Furthermore, they showed that CSF1 inhibitors were found to have a significant anti-angiogenic effect. Importantly, withdrawal of these CSF1 inhibitors did not lead to a rebound effect of tumor growth in tumor bearing mice, whereas removal of anti-VEGF therapy led to a rapid regrowth of murine tumors.

When considering novel therapeutic approaches, it is important to determine the stability of the potential target. Here we show that the CSF1 expression by primary LMS tumor cells is maintained in the majority of metastatic lesions and also in an experimental xenograft model system. Our results suggest that CSF1 may affect tumor angiogenesis (most likely through an attraction of macrophages to the tumor site) and that it may represent a promising therapeutic target to inhibit angiogenesis in nongynecologic LMS.

\section{Acknowledgments}

The authors dedicate this article to the memory of Rebecca Cohen, LMS patient and fundraiser for LMS research.

\section{References}

1. Condeelis J, Pollard JW. Macrophages: obligate partners for tumor cell migration, invasion, and metastasis. Cell 2006, 124:263-266

2. Lewis CE, Pollard JW. Distinct role of macrophages in different tumor microenvironments. Cancer Res 2006, 66:605-612

3. Qian BZ, Pollard JW. Macrophage diversity enhances tumor progression and metastasis. Cell 2010, 141:39-51

4. Frank RE, Saclarides TJ, Leurgans S, Speziale NJ, Drab EA, Rubin DB. Tumor angiogenesis as a predictor of recurrence and survival in patients with node-negative colon cancer. Ann Surg 1995, 222 : 695-699

5. Giatromanolaki A, Koukourakis M, O'Byrne K, Fox S, Whitehouse R, Talbot DC, Harris AL, Gatter KC. Prognostic value of angiogenesis in operable non-small cell lung cancer. J Pathol 1996, 179:80-88

6. Hollingsworth HC, Kohn EC, Steinberg SM, Rothenberg ML, Merino MJ. Tumor angiogenesis in advanced stage ovarian carcinoma. Am J Pathol 1995, 147:33-41

7. Jaeger TM, Weidner N, Chew K, Moore DH, Kerschmann RL, Waldman FM, Carroll PR, Tumor angiogenesis correlates with lymph node metastases in invasive bladder cancer. J Urol 1995, 154:69-71

8. Olivarez D, Ulbright T, DeRiese W, Foster R, Reister T, Einhorn L, Sledge $G$. Neovascularization in clinical stage A testicular germ cell tumor: prediction of metastatic disease. Cancer Res 1994, 54:28002802

9. Weidner N, Carroll PR, Flax J, Blumenfeld W, Folkman J. Tumor angiogenesis correlates with metastasis in invasive prostate carcinoma. Am J Pathol 1993, 143:401-409

10. Weidner N, Semple JP, Welch WR, Folkman J. Tumor angiogenesis and metastasis-correlation in invasive breast carcinoma. $\mathrm{N}$ Engl J Med 1991, 324:1-8

11. Lin EY, Li JF, Gnatovskiy L, Deng Y, Zhu L, Grzesik DA, Qian H, Xue $\mathrm{XN}$, Pollard JW. Macrophages regulate the angiogenic switch in a mouse model of breast cancer. Cancer Res 2006; 66:11238-11246

12. Leek RD, Lewis CE, Whitehouse R, Greenall M, Clark J, Harris AL, Association of macrophage infiltration with angiogenesis and prognosis in invasive breast carcinoma. Cancer Res 1996, 56:4625-4629

13. Onita T, Ji PG, Xuan JW, Sakai H, Kanetake H, Maxwell PH, Fong GH, Gabril MY, Moussa M, Chin JL. Hypoxia-induced, perinecrotic expression of endothelial Per-ARNT-Sim domain protein-1/hypoxia-inducible factor-2alpha correlates with tumor progression, vascularization, and focal macrophage infiltration in bladder cancer. Clin Cancer Res 2002, 8:471-480

14. Lagacé R, Schürch W, Seemayer TA. Myofibroblasts in soft tissue sarcomas. Virchows Arch A Pathol Anat Histopathol 1980, 389:1-11

15. Espinosa I, Beck AH, Lee CH, Zhu S, Montgomery KD, Marinelli RJ, Ganjoo KN, Nielsen TO, Gilks CB, West RB, van de Rijn M. Coordinate expression of colony-stimulating factor-1 and colony-stimulating factor-1-related proteins is associated with poor prognosis in gynecological and nongynecological leiomyosarcoma. Am J Pathol 2009, 174:2347-2356

16. Lee $\mathrm{CH}$, Espinosa I, Vrijaldenhoven S, Subramanian S, Montgomery KD, Zhu S, Marinelli RJ, Peterse JL, Poulin N, Nielsen TO, West RB, Gilks CB, van de Rijn M. Prognostic significance of macrophage infiltration in leiomyosarcomas. Clin Cancer Res 2008, 14:1423-1430

17. Gratzinger D, Zhao S, Marinelli RJ, Kapp AV, Tibshirani RJ, Hammer AS, Hamilton-Dutoit S, Natkunam Y. Microvessel density and expression of vascular endothelial growth factor and its receptors in diffuse large B-cell lymphoma subtypes. Am J Pathol 2007, 170:1362-1369

18. Marinelli RJ, Montgomery K, Liu CL, Shah NH, Prapong W, Nitzberg M, Zachariah ZK, Sherlock GJ, Natkunam Y, West RB, van de Rijn M, 
Brown PO, Ball CA. The Stanford Tissue Microarray Database. Nucleic Acids Res 2008, 36:D871-D877

19. Cheng H, Clarkson PW, Gao D, Pacheco M, Wang Y, Nielsen TO Therapeutic antibodies targeting CSF1 impede macrophage recruitment in a xenograft model of tenosynovial giant cell tumor. Sarcoma 2010, 2010:174528

20. Lee $\mathrm{CH}$, Xue H, Sutcliffe M, Gout PW, Huntsman DG, Miller DM, Gilks $\mathrm{CB}$, Wang YZ. Establishment of subrenal capsule xenografts of primary human ovarian tumors in SCID mice: potential models. Gynecol Oncol 2005, 96:48-55

21. Liu CL, Prapong W, Natkunam Y, Alizadeh A, Montgomery K, Gilks $\mathrm{CB}$, van de Rijn M. Software tools for high-throughput analysis and archiving of immunohistochemistry staining data obtained with tissue microarrays. Am J Pathol 2002, 161:1557-1565

22. Kubota Y, Takubo K, Shimizu T, Ohno H, Kishi K, Shibuya M, Saya H, Suda T. M-CSF inhibition selectively targets pathological angiogenesis and lymphangiogenesis. J Exp Med 2009, 206:1089-1102

23. Olumi AF, Grossfeld GD, Hayward SW, Carroll PR, Tlsty TD, Cunha GR. Carcinoma-associated fibroblasts direct tumor progression of initiated human prostatic epithelium. Cancer Res 1999, 59:50025011
24. Orimo A, Gupta PB, Sgroi DC, Arenzana-Seisdedos F, Delaunay T, Naeem R, Carey VJ, Richardson AL, Weinberg RA. Stromal fibroblasts present in invasive human breast carcinomas promote tumor growth and angiogenesis through elevated SDF-1/CXCL12 secretion. Cell 2005, 121:335-348

25. Saenz NC, Heslin MJ, Adsay V, Lewis JJ, Leung DH, LaQuaglia MP, Brennan MF. Neovascularity and clinical outcome in high-grade extremity soft tissue sarcomas. Ann Surg Oncol 1998, 5:48-53

26. Yudoh K, Kanamori M, Ohmori K, Yasuda T, Aoki M, Kimura T. Concentration of vascular endothelial growth factor in the tumour tissue as a prognostic factor of soft tissue sarcomas. $\mathrm{Br} \mathrm{J}$ Cancer 2001, 84:1610-1615

27. West CC, Brown NJ, Mangham DC, Grimer RJ, Reed MW. Microvessel density does not predict outcome in high grade soft tissue sarcoma. Eur J Surg Oncol 2005, 31:1198-1205

28. DuBois $S$, Demetri G. Markers of angiogenesis and clinical features in patients with sarcoma. Cancer 2007, 109:813-819

29. Allavena P, Sica A, Garlanda C, Mantovani A. The Yin-Yang of tumorassociated macrophages in neoplastic progression and immune surveillance. Immunol Rev 2008, 1:155-161 